\title{
Fear contagion in zebrafish: a behaviour affected by familiarity
}

2

3

5

6

7

8 *Corresponding author: email, postal address and telephone

$9 \quad$ c.garciadeleaniz@swansea.ac.uk

10 Centre for Sustainable Aquatic Research (CSAR), Department of Biosciences, Swansea

11 University, UK.

$12 \quad$ Tel +44 (0)1792295383

13

14

15

16

17

18

19

20

21

22

23

24

25 


\section{Abstract}

27 Emotional contagion has recently been described in fish but whether it is affected by

28 familiarity is not known. We tested whether the sight of a distressed conspecific elicited fear

29 in zebrafish, and whether this was modulated by familiarity. Groups of six zebrafish were

30 housed together in the same tanks for 7 days to create familiar conditions. The behaviour of

31 individual fish was then recorded in paired tanks within sight of either a familiar or an

32 unfamiliar individual, before and after distilled water or an alarm substance was added to the

33 demonstrator, but not to the observer. As expected, addition of distilled water did not elicit

34 any behavioural change in either the demonstrator or the observer. However, addition of an

35 alarm cue triggered anti-predatory behaviours in the demonstrator which caused the

36 expression of anti-predatory behaviours in the observer, suggesting the existence of fear

37 contagion. Furthermore, the extent of fear contagion was affected by familiarity, and

38 observers were more active, swam closer to the bottom and further away from the

39 demonstrator when they watched a distressed familiar neighbour than when they watched an

40 unfamiliar fish. Our results have implications for fish welfare because they show that fish can

41 become stressed by simply watching others become stressed. They also have implications for

42 experimental design because fish housed in separate tanks cannot be assumed to be

43 statistically independent if they can eavesdrop on their neighbours.

45 Key-words: emotional contagion; empathy; alarm response; animal model 


\section{Introduction}

52 Emotional contagion can be defined as the instantaneous matching of emotional state

53 between an observer and a demonstrator (Nakahashi \& Ohtsuki, 2018). This phenomenon has

54 been explained through the perception-action mechanism, which postulates that the

55 perception of a demonstrator's state triggers a neural, unconscious and automatic

56 representation of the same state in the observer, causing an equivalent expression of

57 behaviours (Preston \& de Waal, 2002). Emotional contagion is considered a component and evolutionary precursor of empathy (Preston \& de Waal, 2002) and has been demonstrated in humans, birds and mammals (Gonzalez-Liencres et al., 2014; Reimert et al., 2014). fear in rodents is freezing behaviour, which can be triggered by a mild electric shock (Lezak et al., 2017; Pisansky et al., 2017). Fear elicited in this way propagates from frightened demonstrators to naïve observers, resulting in increased frequency of freezing (Jeon et al., 2010; Knapp et. al, 2007; Knapska et al., 2006) and activation of the amygdala in the observer (Knapska et al., 2006). Moreover, it seems that how an emotion is shared between individuals is modulated by contextual aspects such as kinship, familiarity and social closeness (Liévin-Bazin et al., 2018; Preston \& de Waal, 2002). For instance, when mice are paired with distressed demonstrators they tend to freeze if they had been reared together, but become more active if they come from different cages (Gonzalez-Liencres et al., 2014). In addition, pain perception in mice is more intense when observers are familiar with demonstrators than when they are strangers (Langford et al., 2006). Observers typically respond differently to signals sent by familiar and unfamiliar conspecifics (Gonzalez-

74 Liencres et al., 2014; Jeon et al., 2010), a strategy thought to be adaptive as it can help avoid

75 sensory overload (Hutchinson, 2005) and focuses attention on signals emitted by those 
76 neighbours that matter the most, including 'nasty neighbours' and 'dear enemies' (Müller \&

77 Manser, 2007).

78 A recent study has provided evidence for fear contagion in zebrafish (Oliveira et al.,

79 2017), which suggests that this phenomenon may be conserved among social vertebrates.

80 However, to what extent fear contagion in fishes can be affected by the degree of familiarity

81 between demonstrators and observers is not known. Familiarity can broadly be defined as the

82 ability to discriminate between individuals based on previous interactions, and is influenced

83 by the time of interaction and the size of the group among fishes (Griffiths, 2003).

84 Here we used dyadic behavioural tests to assess if fear contagion was affected by

85 familiarity in zebrafish. To this end, demonstrators were exposed to either distilled water or

86 an alarm substance, known to cause a fear response on this species (Kalueff et al., 2013).

87 Zebrafish are highly social (Gerlai, 2010), can discriminate familiar from unfamiliar fish after

88 only 20 min of interaction (Hinz et al., 2013; Madeira \& Oliveira, 2017), and form cohesive

89 groups when under threat (Speedie \& Gerlai, 2008). Therefore, we hypothesized that

90 observers would show a heightened fear response to the sight of distressed demonstrators

91 when they had been reared together (i.e. were familiar), than when they had been reared apart

92 (i.e. were unfamiliar). 


\section{$94 \quad$ Methods \& Materials}

95 Experimental fish and husbandry conditions

96 Two month old, laboratory-reared zebrafish (Danio rerio) of homogeneous size were sourced

97 from a local supplier and kept in four $50 \mathrm{~L}$ tanks (density $=2$ fish/L) connected to a

98 recirculation system for four months before testing. Water quality was maintained by

99 mechanical, biological and chemical filtration, in addition to UV disinfection. Water

100 temperature was kept at $28 \pm 1^{\circ} \mathrm{C}, \mathrm{pH}$ at 7.2 and ammonia and nitrite at recommended

101 optimal levels for the species. Photoperiod was set at 12D:12L with the help of fluorescent

102 lights (150 lumens) with the start of the light phase set at 7:00 hrs. Fish were fed commercial

103 pellets twice a day (Nutricom Pet, $38 \%$ protein, $4 \%$ lipids) and Artemia salina once daily.

Development of familiar and unfamiliar groups

106 Seven days prior to testing, a sample of 156 adults of both sexes was collected haphazardly

107 from the four stock tanks, mixed, and allocated at random in groups of six to $26 \mathrm{x} 20 \mathrm{~L}$ glass aquaria (40L x $20 \mathrm{~W} \times 25 \mathrm{H} \mathrm{cm})$. Aquaria were filled with system water, were fitted with a sponge filter to maintain water quality, and had their bottom and sides covered with white plastic sheets to prevent visual contact with others groups. Food was offered twice a day, as

111 above.

\section{Acclimation period}

114 After seven days of being reared in groups of six, two individuals from either the same or different aquaria (i.e. familiar or unfamiliar conditions) were transferred to two $2 \mathrm{~L}$ test aquaria $(20 \mathrm{~L}$ x $10 \mathrm{~W}$ x $20 \mathrm{H} \mathrm{cm})$ placed side by side and left to acclimatize for 18 hours prior to testing, one fish serving as a 'demonstrator' and the other as an 'observer'. To ensure that unfamiliar fish would not become 'familiar' during the acclimation period, a removable 
119 divider was placed between the two test aquaria, so that demonstrator and observer had no

120 visual contact until the divider was lifted just prior to testing. Similarly, and in order to avoid

121 a potential disruption of familiar dyads, these were acclimated without dividers, in full sight

122 of each other. To test if this could have affected their subsequent behaviour, we tested 8

123 additional familiar dyads acclimatized with dividers, and compared their behaviour to

124 familiar dyads acclimatized without dividers.

125

Testing of fear contagion

127 Following the $18 \mathrm{~h}$ acclimation period, the divider preventing visual contact was removed and

128 the demonstrator and observer were simultaneously recorded (Sony DCR-SX45 Digital VCR)

129 for 5 min (basal behaviour). A syringe connected to a small silicon tube was then used to

130 remotely deliver $2 \mathrm{ml}$ of either distilled water or an alarm substance to the demonstrator

131 (delivery being allocated at random), and their behaviours were recorded at 10 minute

132 intervals over an hour, as shown schematically in Figure 1. To obtain the alarm substance,

133 one zebrafish from the stock tanks was euthanized by an overdose of clove oil ( $2 \mathrm{ml} / \mathrm{L}$ water),

134 and c. $1 \mathrm{~cm}^{2}$ of skin from each flank was removed, macerated in $10 \mathrm{ml}$ of distilled water and

135 filtered. Fresh alarm substance was prepared every morning before testing.

136 We tested 48 dyads exposed to alarm substance (24 unfamiliar and 24 familiar) and 20

137 dyads exposed to distilled water (10 unfamiliar and 10 familiar). In addition, 20

138 demonstrators (10 exposed to alarm substance and 10 exposed to distilled water) were tested

139 without observers to ascertain which behaviours were modified by the addition of the alarm

140 substance, and to what extent the presence of observers influenced the demonstrator's

141 behaviour. Fish were only used once in the experiments, either as observers or demonstrators.

142 The $10 \mathrm{ml}$ of alarm substance obtained from one fish was enough to test five dyads, thus for

143 all the experiments 12 zebrafish were used for the preparation of alarm substance. 
bioRxiv preprint doi: https://doi.org/10.1101/521187; this version posted January 17,2019 . The copyright holder for this preprint (which was not certified by peer review) is the author/funder, who has granted bioRxiv a license to display the preprint in perpetuity. It is made available under aCC-BY-NC-ND 4.0 International license.

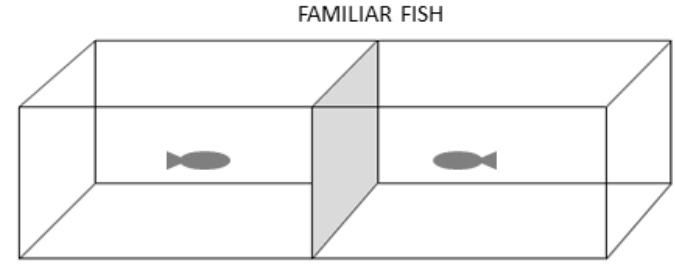

UNFAMILIAR FISH familiar and unfamiliar zebrafish dyads. 
162 We used ZebTrack (Pinheiro-da-Silva et al., 2016) to extract from the video recordings six

163 behavioural metrics that had previously been shown to describe well the stress response of

164 zebrafish (Gerlai et al, 2008; Luca \& Gerlai, 2012; Tran \& Gerlai, 2013), namely : (1) mean

165 swimming speed, (2) maximum swimming speed, (3) total distance travelled, (4) time spent

166 freezing, (5) swimming depth (i.e. distance from the tank bottom) and (6) mean distance to

167 the conspecific's tank.

\section{Statistical Analysis}

Statistical analysis was conducted in R v. 3.4 .3 (R Core Team 2013). Our experiment conformed to a fully factorial $2 \times 2 \times 2$ BACI design (before-after-control-impact) and we modelled the behaviour of the observer (dependent variable) as a linear mixed effect model using the lme4 (Bates et al., 2014) and lmerTest (Kuznetsova et al., 2017) R packages. We used as fixed effects (predictors) the behaviour of the demonstrator, the time (before or after the stressor was added), the familiarity (familiar vs unfamiliar dyad) and the stressor type (alarm substance vs distilled water), and considered the dyad identity as a random effect to control for variation among test arenas and account for potential non-independence of observations. For each behavioural metric, we started with a maximal model with all main effects and interactions and used the step and dredge functions in the MuMIn package (Bartoń, 2013) to arrive at a minimal adequate model via Maximum Likelihood on the basis of single deletion tests and relative changes in AICc values. The most plausible model was refitted by REML and the model adequacy and assumptions were checked by examining plots of fitted vs residuals, fitted vs observed values, as well as plots of random effects and standardized fixed effects using the sjPlot package (Lüdecke, 2016). We report all models 
187 To better assess the extent to which observers were able to match the overall behaviour of

188 demonstrators, we also carried out a principal components analysis and modelled the scores

189 along the first two first principal components (which together explained $96 \%$ of variation) as

190 a function of fish type (observer or demonstrator) and degree of familiarity.

192 Ethical note

193 All experimental procedures were authorized by the Animal Ethics Committee permit CEUA

194 042/2015 and Animal Welfare and Ethical Review Body permit IP-1516-8. 


\section{Results}

197

198

199

200

201

202

203

204

205

Response to alarm cues (single tests)

Inspection of temporal data (Supplementary Material, Figure S1) indicated that the response of single fish to alarm cues was rapid and did not persist for more than 10 minutes after the administration of the alarm substance (probably due to habituation). We, therefore, concentrated the analysis on the first 10 minutes after addition of the stimuli. Compared to baseline values, the addition of an alarm cue resulted in an increase in average swimming speed and time spent freezing that was not observed when distilled water was added (average speed, before-after $=6.20, \mathrm{SE}=0.81, t_{17}=7.68, P<0.001$; blank-alarm $=7.08, \mathrm{SE}=1.11$, $t_{27.19}=6.36, P<0.001 ;$ after $\mathrm{x}$ alarm $=-6.39, \mathrm{SE}=1.11, t_{17}=-5.75, P<0.001$; time spent freezing, before-after $=118.7, \mathrm{SE}=13.24, t_{18}=8.97, P<0.001$; blank-alarm $=110.6, \mathrm{SE}=$ $13.52, t_{35.94}=8.18, P<0.001$, after $\mathrm{x}$ alarm $\left.=-105.56, \mathrm{SE}=18.72, t_{18}=-5.64, P<0.001\right)$. Fish also stayed closer to the bottom after alarm cue or distilled water were added (beforeafter $\left.=-3.50, \mathrm{SE}=1.00, t_{18}=-3.49, P=0.002\right)$, but no significant change was detected in relation to maximum speed or distance travelled $(P>0.05$; Supplementary Material, Figure S1) and these were not considered any further. Results also indicated that whether familiar demonstrators were visually isolated or not during the $18 \mathrm{hrs}$ of acclimation did not affect their subsequent behaviours (Supplementary Material, Table S1).

\section{Fear Contagion from Demonstrators to Observers (dyadic tests)}

\section{Swimming Speed}

As expected from the single tests above, demonstrators in the dyadic tests increased their average swimming speed when an alarm substance was added, but not when distilled water was added (Figure 2). Observers responded by increasing their speed when the demonstrator was unfamiliar, but by decreasing it when the demonstrator was familiar (Figure 2; estimate 
221 demonstrator $=0.40, P=0.002$; estimate time $=0.93, P<0.001$; estimate familiarity $\mathrm{x}$ time $=$

$2221.18, P<0.001$; estimate time $\mathrm{x}$ stressor $=0.53, P=0.002$; estimate familiarity $\mathrm{x}$ time $\mathrm{x}$ stressor $=-0.60, P<0.001)$. Fear contagion, hence, was affected by familiarity.

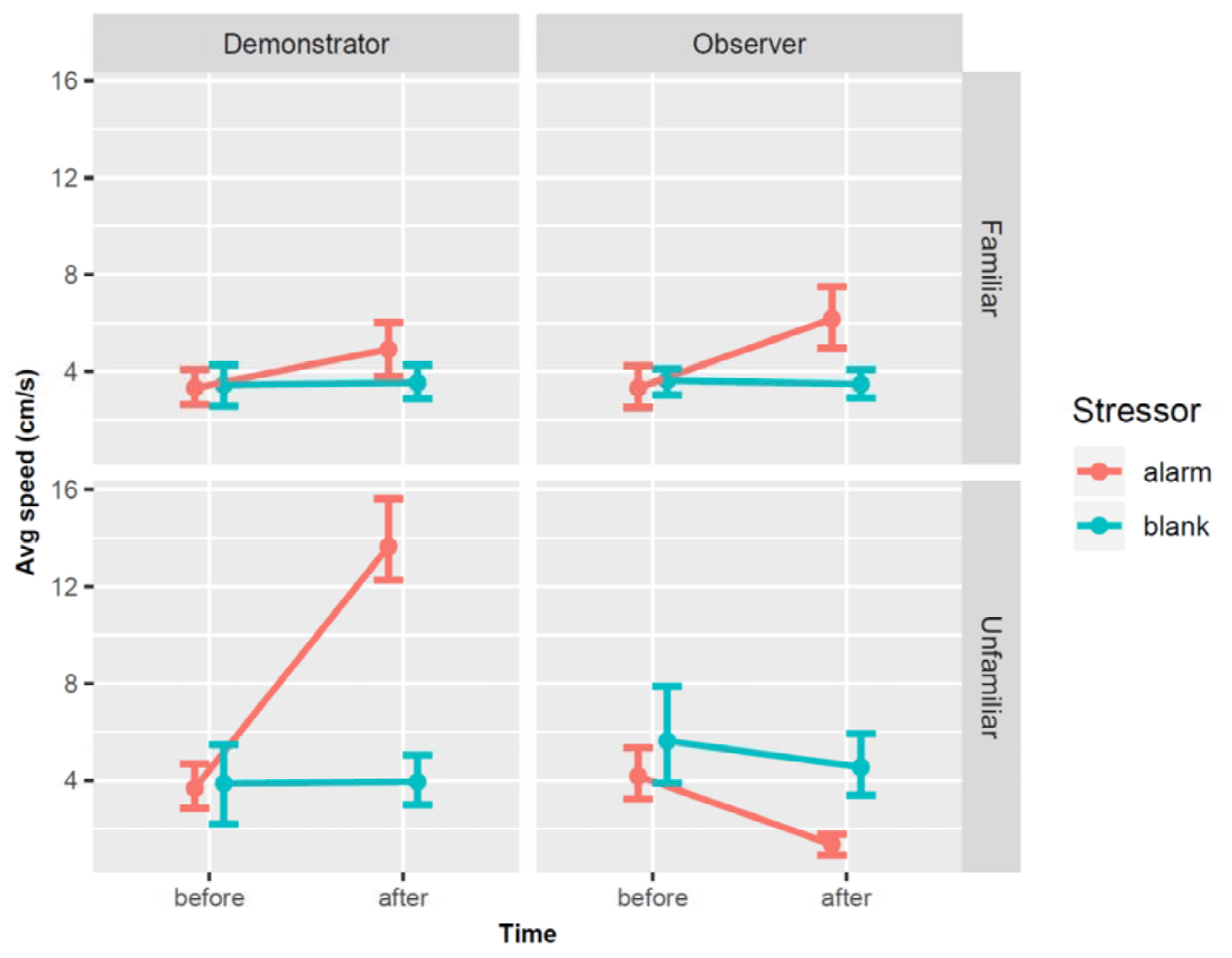

226 Figure 2. Changes in the swimming speed (mean \pm 95CI) of zebrafish before (basal) and after distilled water or an alarm cue were delivered to the demonstrator in dyadic tests 
237 As expected also from the single tests above, demonstrators spent more time freezing when

238 an alarm substance was added, but not when distilled water was added (Figure 3). The time

239 observers spent freezing increased when an alarm substance was added to the demonstrator

240 (estimate $=0.85, P<0.001$ ), and also with time (estimate $=0.90, P<0.001$ ), but was

241 negatively correlated with the time spent freezing by the demonstrators (estimate $=-0.19, P=$

242 0.024). There was a significant interaction between time and stressor (estimate $=0.63, P<$

243 0.001) as observers only increased the time spent freezing over basal values when the alarm

244 substance was added, not when distilled water was added. Familiarity, hence, did not

245 influence the freezing response, which was very strong under both conditions.

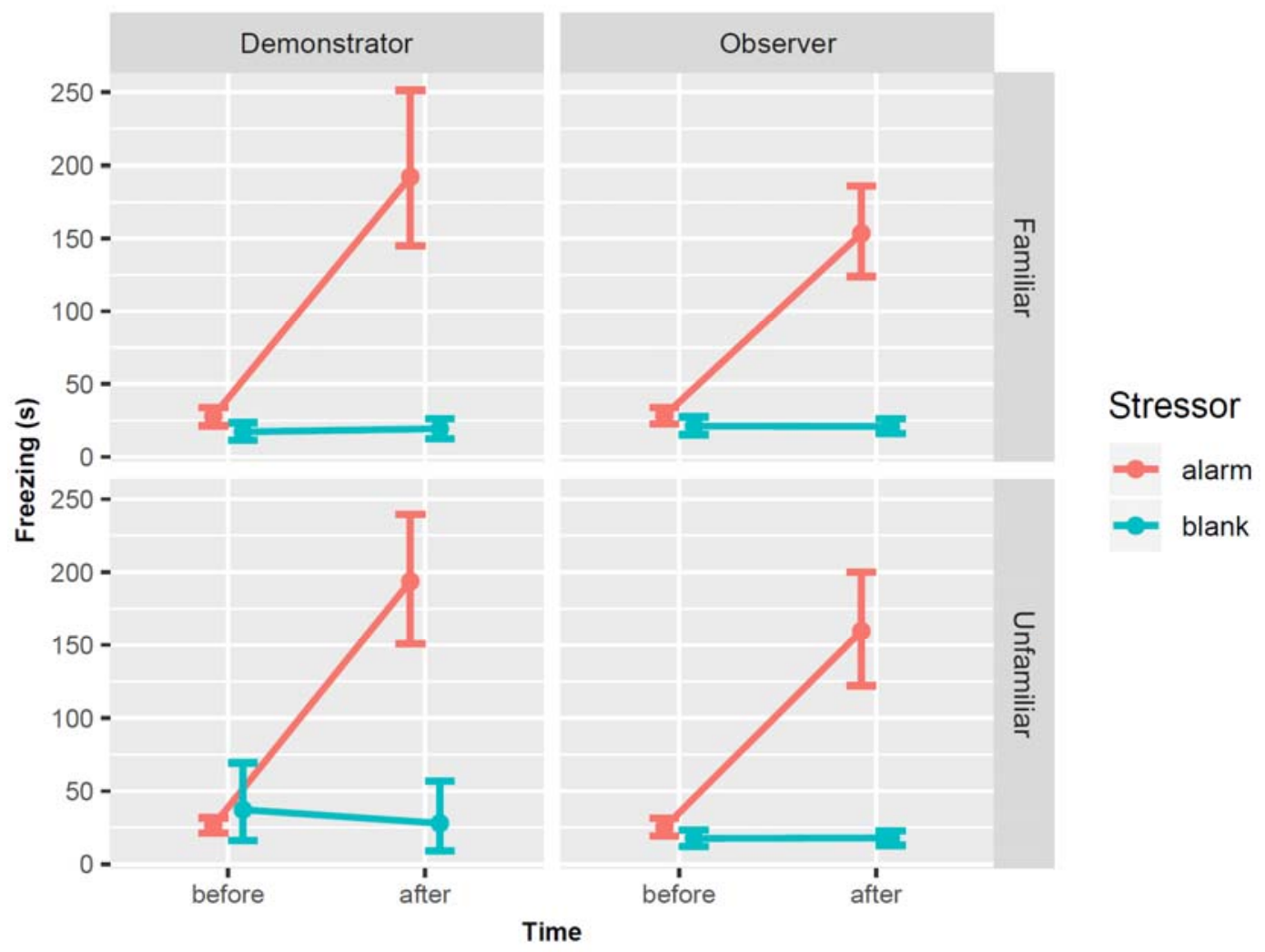

246 Figure 3. Changes in the freezing behaviour (mean $\pm 95 \mathrm{CI}$ ) of zebrafish before (basal) and 247 after distilled water or an alarm cue were delivered to the demonstrator in dyadic tests 248 involving familiar and unfamiliar pairs. 
253 Following the addition of the alarm substance, demonstrators moved closer to the bottom of

254 the tank, a behaviour not seen when distilled water was added. Observers mimicked this

255 behaviour (Figure 4), tracking what the demonstrator did (estimate $=0.19, P=0.033$ ).

256 Swimming depth increased over basal values (estimate $=0.23, P=0.011$ ), as well as with the

257 addition of the alarm cue (estimate $=0.31, P=0.018$ ). There were significant interactions

258 between demonstrator's depth and familiarity (estimate $=0.25, P=0.03$ ), demonstrator's

259 depth and time (estimate $=0.25, P=0.002$ ), familiarity and time (estimate $=0.44, P<$

260 0.001), familiarity and stressor (estimate $=0.54, P<0.001$ ), and familiarity $\mathrm{x}$ time $\mathrm{x}$ stressor

261 (estimate $=0.28, P=0.003$ ). There was, hence, evidence of fear contagion which was also

262 affected by familiarity.

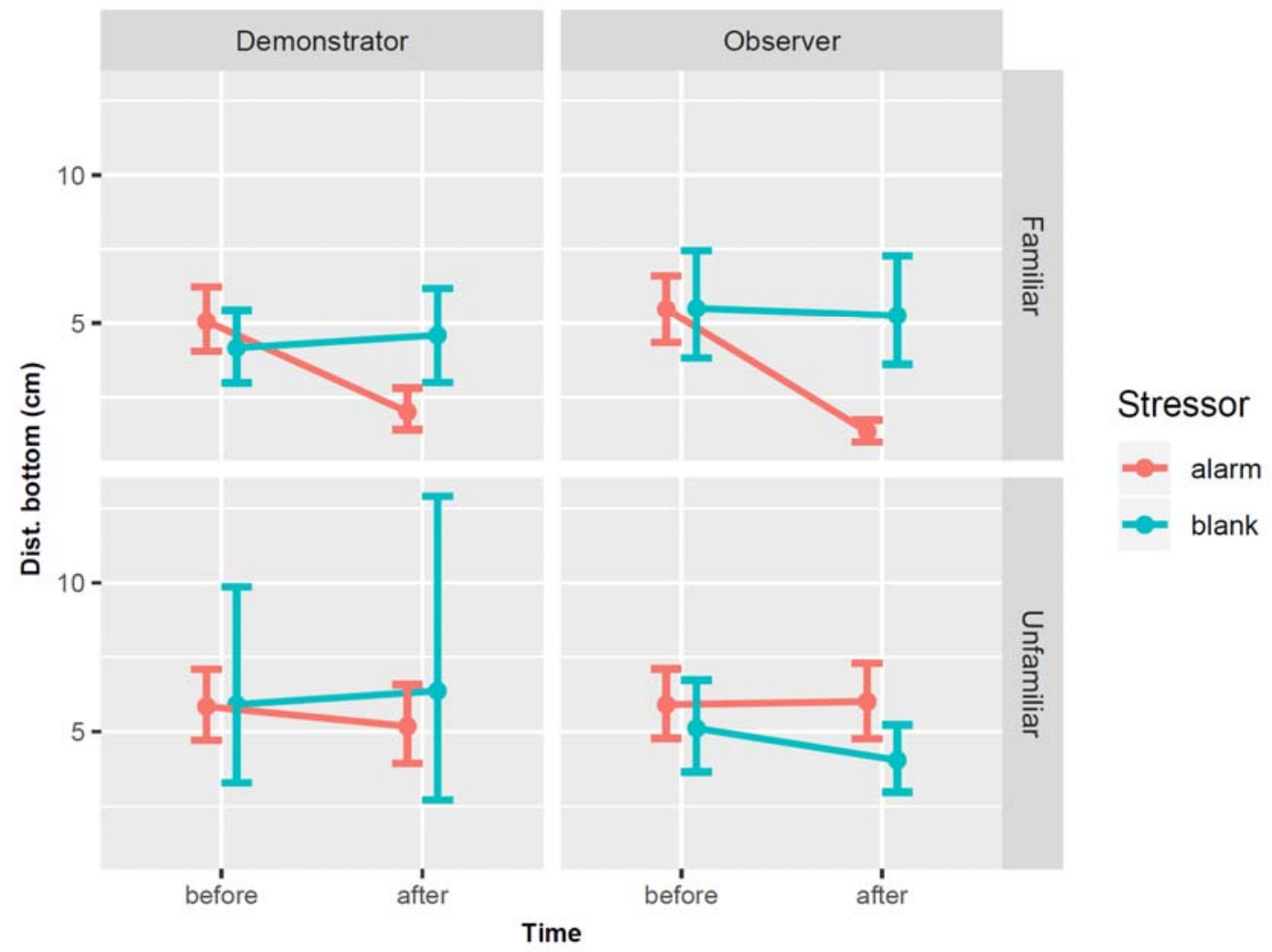

263 Figure 4. Changes in the swimming depth (mean $\pm 95 \mathrm{CI}$ ) of zebrafish before (basal) and 264 after distilled water or an alarm cue were delivered to the demonstrator in dyadic tests 265 involving familiar and unfamiliar pairs.

Distance to the conspecific's tank (proximity to the demonstrator) 
269 Following the addition of the alarm substance to the demonstrator, the observer swam closer

270 to the demonstrator's tank (Figure 5, estimate $=0.60, P<0.001$ ), something that did not

271 happen when distilled water was added. Distance to the demonstrator decreased over basal

272 values (estimate $=0.43, P=0.005)$ but increased with familiarity (estimate $=0.16, P=0.031)$

273 and was also affected by the interactions between demonstrator and time (estimate $=0.36, P$

$274=0.03$ ), and between time and stressor (estimate $=-0.49, P<0.001$ ). There was thus

275 evidence of fear contagion with respect to proximity to the other fish's tank and this also

276 seemed to be influenced by familiarity.

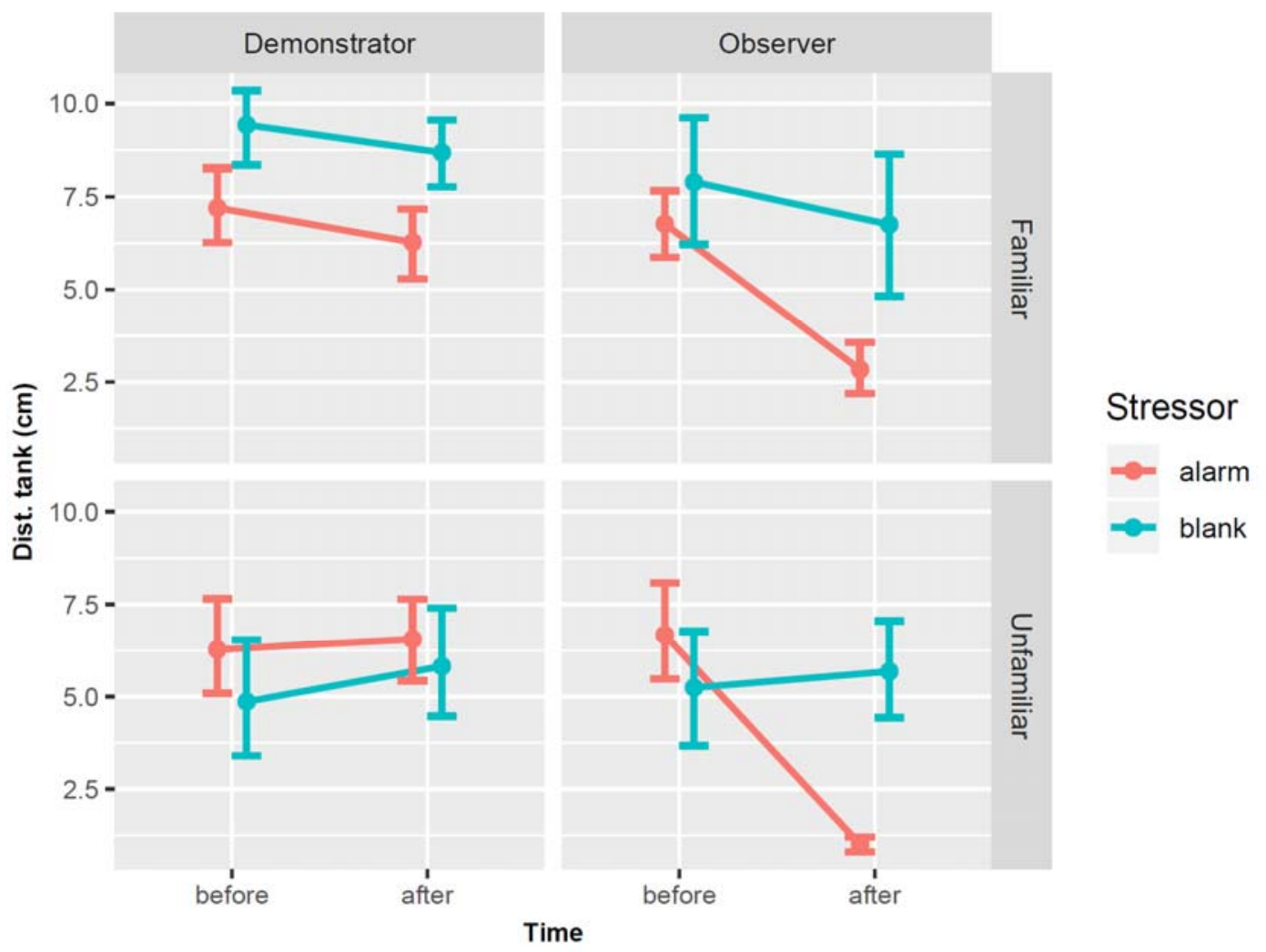

277 Figure 5. Changes in the distance to the conspecific's tank (mean $\pm 95 \mathrm{CI}$ ) of zebrafish before 278 (basal) and after distilled water or an alarm cue were delivered to the demonstrator in dyadic 
284 PCA analysis showed that the behaviour of demonstrators and observers was similar and

285 showed little variation among individuals when the fish were not stressed (i.e. when distilled

286 water was added or before an alarm cue was added, Figure 6a-c, Figure 6e-g). The first

287 component, PC1, accounted for $89.8 \%$ of the variation and described freezing behaviour,

288 while PC2 accounted for 5.9\% of the variation and described variation in swimming speed

289 and proximity to the conspecific. No statistical difference between observer and

290 demonstrators was found along PC1 $\left(F_{3,90}=0.847, P=0.472\right)$, but there was a marked

291 difference along PC2 $\left(F_{3,90}=68.52, P<0.001\right.$; Figure 6d, Figure 6h $)$ which depended on the

292 type of fish (estimate for observer $=1.21, \mathrm{SE}=0.64, P=0.009$ ), the extent of familiarity

293 (estimate for unfamiliar $=-7.72, \mathrm{SE}=0.91, P<0.001$ ), and their interaction (estimate for

294 observer $\mathrm{x}$ unfamiliar $=15.44, \mathrm{SE}=1.29, P<0.001)$. Thus, observers were better able to

295 mimic the anti-predatory behaviour of demonstrators when they were familiar than when they

296 were unfamiliar (Figure 6d). 
300

301

302

303

304

305

306

307

308

309

310

311

312

313

314

315

316

317

318

319

320

321

322

323

324

325

326

327

328

329

330

331

332

333

334

335

336

337

338

339

340

341

342

343

344

345

346

347

348

349
Familiar
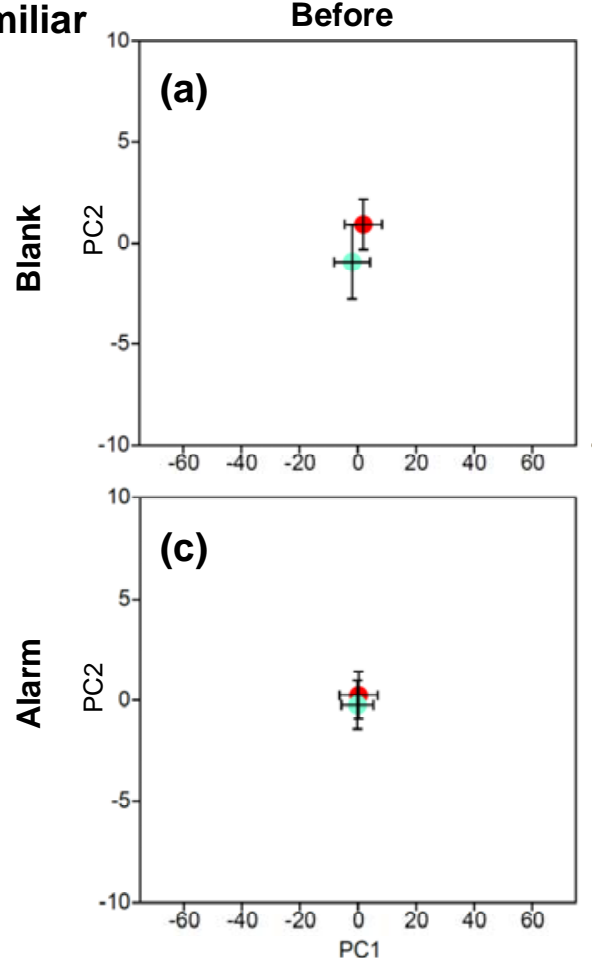

Unfamiliar
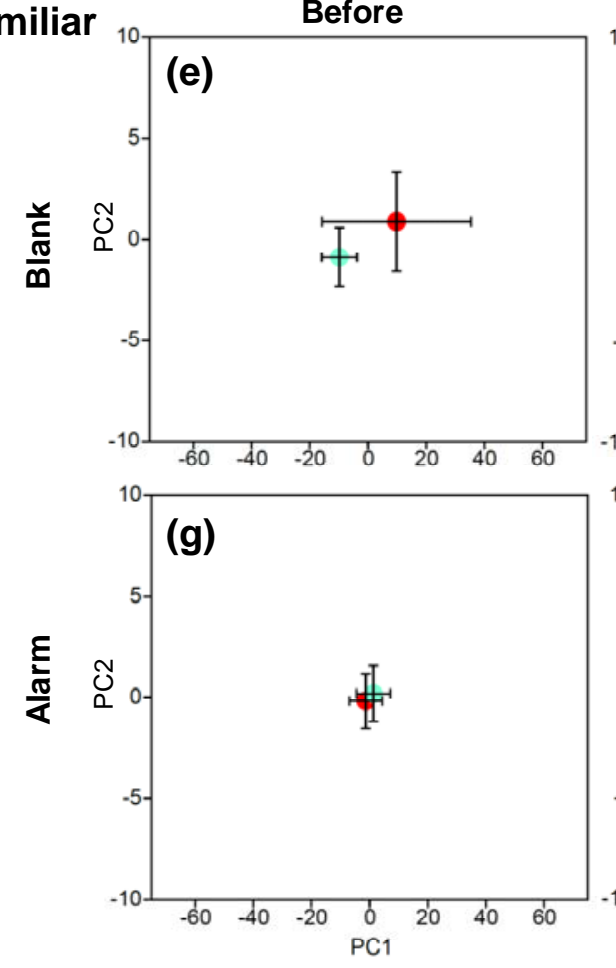
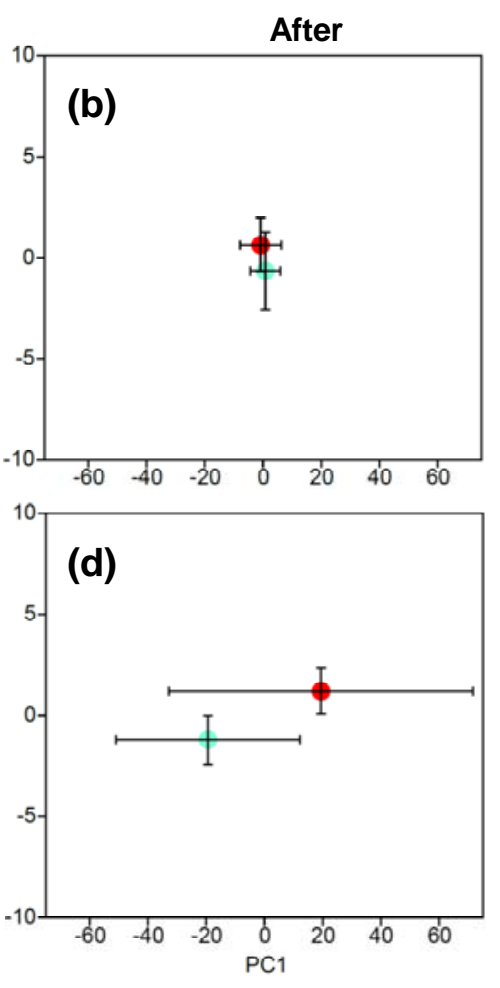

After
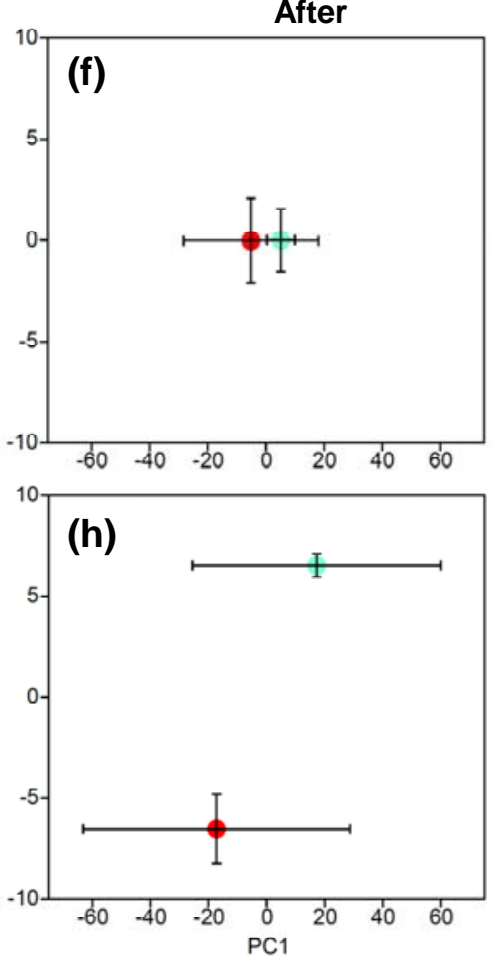

Figure 6. Variation along the first two principal components (means $\pm 95 \mathrm{CI}$ ) describing the behaviour of familiar and unfamiliar dyads of zebrafish before (basal) and after distilled water or an alarm cue were delivered to the demonstrator (red) but not to the observer (green). 
350

351

352

353

354

\section{DISCUSSION}

Our study provides novel evidence in support for the existence of fear contagion in fish, and suggests that this is influenced by familiarity, as shown for mammals (Gonzalez-Liencres, et al 2014; Jeon et al., 2010). The alarm response of zebrafish has been characterised by an increase in swimming speed interspersed with freezing bouts and diving to the bottom (Kalueff et al., 2013). To trigger a fear response we added an alarm substance to the water, schreckstoff, (von Frisch, 1938), a well-established stressor for zebrafish (Jesuthasan \& Mathuru, 2008). As expected, the addition of the alarm substance triggered a flight response in zebrafish, which swam faster, moved to deeper waters, and included bouts of freezing behaviour, something not observed when distilled water was added. Such anti-predatory behaviours were mimicked to some extent by the observers, even though they had no direct exposure to the stressor. Thus, the mere sight of a distressed conspecific was enough to trigger in the observer the same fear response experienced and displayed by the demonstrator.

Our study shows that fear contagion is modulated by familiarity in zebrafish, since three of the four responses examined varied depending on whether the observers were watching individuals they were familiar with. Compared to unfamiliar demonstrators, observers responded to the sight of familiar demonstrators by matching their swimming speeds more closely, and by moving closer to the bottom. Observers also moved closer to the demonstrator at the sight of a distressed conspecific, but - perhaps unexpectedly, this was more pronounced when they were paired with unfamiliar demonstrators. These results add support to evidence indicating that fish are capable of identifying and reacting to the behaviour of conspecifics (Jesuthasan \& Mathuru, 2008; Rey et al., 2015). In social species, fear contagion is thought to be adaptive as it will often increase the probability of escaping from predators (Nakahashi \& Ohtsuki, 2018). 
376 (Chivers et al. 1995; Lachlan et al. 1998) and facilitates social learning (Swaney et al., 2001),

377 and our study shows that familiarity also affects fear contagion, which may explain why

378 association with familiar fish is generally adaptive (Griffiths, 2003). For example, familiar

379 brown trout respond $14 \%$ faster than unfamiliar fish to a predator attack (Griffiths et al.

380 2004), most likely because they can interpret signals from familiar fish more accurately.

381 Familiarity in our study was established rapidly, after only seven days of cohabitation, which

382 is consistent with previous results in zebrafish (Madeira \& Oliveira, 2017) and other species

383 (Griffiths, 2003), where individuals were able to recognize familiar neighbours after short

384 periods of interaction. Although it is possible that some individuals in our study may have

385 become familiar in the stock tanks (i.e., before the 7 day cohabitation experiment), this is

386 unlikely as the group size was too large (100 fish/tank) for individual recognition (Griffiths \&

387 Magurran, 1997), fish were mixed and allocated at random to 26 groups, and this would not explain why familiar and unfamiliar fish behaved so differently.

The advantages of familiarity may be accrued through visual recognition, but also through chemical cues (Griffiths, 2003), as fish can recognize the metabolites of conspecifics released in the water (Ward et al. 2009). Zebrafish can use both chemical and visual cues for individual recognition (Hinz et al., 2013), but our study indicates that visual signals alone are enough to trigger fear contagion, as familiar fish behaved differently from unfamiliar fish when no chemical signals were exchanged between observers and demonstrators. The strong shoaling behaviour of zebrafish may help explain the evolution of fear contagion on this species. Unlike social learning, which is thought to have evolved to facilitate the long-term transmission and storage of information (Brown \& Laland, 2003), fear contagion may have evolved to deal with rapid, short-term signals and swift responses, such as the anti-predatory response (Nakahashi \& Ohtsuki, 2015). 
400 It has been proposed that behavioural contagion should be heightened when the

401

402

403 demonstrator displays abnormal or extreme behaviours (Nakahashi \& Ohtsuki, 2015). Freezing is an extreme behaviour that can be induced by alarm substances and is commonly seen in many fish species in response to predators (Gerlai et al. 2000; Gerlai \& Csányi 1990; Miklosi el al. 1997; Roberts et al. 2011; Roberts \& Garcia de Leaniz, 2011). In this sense, the increased duration in freezing bouts following exposure to the alarm substance was highly contagious in our study, but it was not affected by familiarity. We suggest that for zebrafish, freezing behaviour constitutes a more robust signal of danger than bottom dwelling or an increase in swimming speed. Hence, it may be adaptive for an individual to freeze when another one is freezing, regardless of the sender's identity. On the other hand, changes in swimming speed or in the position in the water column form part of the normal behaviour of zebrafish (Kalueff et al., 2013) and may represent less extreme, and hence less reliable, signals.

Our results show that zebrafish can not only distinguish between familiar and unfamiliar conspecifics by visual cues alone, but that they can also eavesdrop on their neighbours living in separate tanks and adjust their behaviour accordingly. This may have implications for fish welfare if, for example, fish can become stressed simply by watching their neighbours become stressed. In the wild, eavesdropping may be adaptive as it allows zebrafish to acquire information on predatory threat from shoal neighbours (Abril-De-Abreu et al., 2015; Oliveira et al., 2017), but the implications for fish welfare in captivity deserve further attention. In livestock, contagion of negative emotions such as fear and anxiety can impair the behaviour and health of the group (Reimert et al., 2013), and our study suggests that the same could happen in zebrafish.

Our results also have implications for experimental design because fish housed in separate tanks may not be assumed to be statistically independent (Colegrave \& Ruxton, 
425 2017) if their behaviour is affected by that of others. Visual isolation of tanks, therefore, must

426 be ensured to prevent eavesdropping. Ultimately, our study indicates that fish are capable of

427 recognizing and mimicking individual behaviours and place them into the right context.

428 Future studies might benefit from investigating if behavioural contagion also occurs in

429 relation to positive stimuli (such as access to food, mates, or enriched habitats) as this could

430 perhaps be used to improve welfare.

431

432 Declaration of interests: none

433 ACKNOWLEDGEMENTS

434 We are grateful to Maria E Leite and Julia Ruiz-Oliveira for assisting with the collection of 435 data. This study was funded by a Royal Society Newton Mobility grant to Prof Sonia 436 Consuegra, ACL, PFS and CGL and the ERDF SMARTAQUA Operation. 
439

440

441

442

443

\section{REFERENCES}

Abril-De-Abreu, R., Cruz, J., \& Oliveira, R. F. (2015). Social eavesdropping in zebrafish: tuning of attention to social interactions. Scientific Reports, 5, 1-14. https://doi.org/10.1038/srep12678

Bartoń, K. (2013). MuMIn-package: Multi-model inference, version 1.9.0

Bates, D., Mächler, M., Bolker, B., \& Walker, S. (2014). Fitting linear mixed-effects models using lme4. ArXiv Preprint ArXiv:1406.5823.

Brown, C., \& Laland, K. N. (2003). Social learning in fishes: a review. Fish and Fisheries, 4(3), 280-288.

Carnevali, L., Montano, N., Statello, R., Coudé, G., Vacondio, F., Rivara, S., ... Sgoifo, A. (2017). Social stress contagion in rats: behavioural, autonomic and neuroendocrine correlates. Psychoneuroendocrinology, 82, 155-163.

Chivers, D. P., Brown, G. E., \& Smith, R. J. F. (1995). Familiarity and shoal cohesion in fathead minnows (Pimephales promelas): implications for antipredator behaviour. Canadian Journal of Zoology, 73(5), 955-960.

Colegrave, N., \& Ruxton, G. D. (2017). Using biological insight and pragmatism when thinking about pseudoreplication. Trends in Ecology \& Evolution.

Gerlai, R. (2010). High-throughput behavioral screens: the first step towards finding genes involved in vertebrate brain function using zebrafish. Molecules, 15(4), 2609-2622.

Gerlai, R., Ahmad, F., \& Prajapati, S. (2008). Differences in acute alcohol $\square$ induced behavioral responses among zebrafish populations. Alcoholism: Clinical and Experimental Research, 32(10), 1763-1773. 
461 Gerlai, R., \& Csányi, V. (1990). Genotype-environment interaction and the correlation structure of behavioral elements in paradise fish (Macropodus opercularis). Physiology \& Behavior, 47(2), 343-356.

Gerlai, R., Lahav, M., Guo, S., \& Rosenthal, A. (2000). Drinks like a fish: zebra fish (Danio rerio) as a behavior genetic model to study alcohol effects. Pharmacology Biochemistry

Hutchinson, J. M. C. (2005). Is more choice always desirable? Evidence and arguments from 
483 Jeon, D., Kim, S., Chetana, M., Jo, D., Ruley, H. E., Lin, S.-Y., ... Shin, H.-S. (2010).

484 Observational fear learning involves affective pain system and Cav1.2 Ca2+ channels in $485 \quad$ ACC. Nature Neuroscience, 13(4), 482-488.

Jesuthasan, S. J., \& Mathuru, A. S. (2008). The alarm response in zebrafish: innate fear in a vertebrate genetic model. Journal of Neurogenetics, 22(3), 211-228.

Kalueff, A. V, Gebhardt, M., Stewart, A. M., Cachat, J. M., Brimmer, M., Chawla, J. S., ... Schneider, H. (2013). Towards a comprehensive catalog of zebrafish behavior 1.0 and

Knapp, D. J., Overstreet, D. H., \& Breese, G. R. (2007). Baclofen Blocks Expression and Sensitization of Anxiety $\square$ Like Behavior in an Animal Model of Repeated Stress and Ethanol Withdrawal. Alcoholism: Clinical and Experimental Research, 31(4), 582-595. preferences and social learning of foraging information in guppies. Animal Behaviour, as Evidence for Empathy in Mice. Science, 312(5782), 1967-1970. https://doi.org/10.1126/science.1128322 
505 Lezak, K. R., Missig, G., \& Carlezon Jr, W. A. (2017). Behavioral methods to study anxiety

506 in rodents. Dialogues in Clinical Neuroscience, 19(2), 181-191. Retrieved from

507 https://www.ncbi.nlm.nih.gov/pubmed/28867942

508 Liévin-Bazin, A., Pineaux, M., Clerc, O., Gahr, M., von Bayern, A. M. P., \& Bovet, D.

509 (2018). Emotional responses to conspecific distress calls are modulated by affiliation in $510 \quad$ cockatiels (Nymphicus hollandicus). PloS One, 13(10), e0205314.

511 Luca, R. M., \& Gerlai, R. (2012). Animated bird silhouette above the tank: acute alcohol

512 diminishes fear responses in zebrafish. Behavioural Brain Research, 229(1), 194-201.

513 Lüdecke, D. (2016). sjPlot: data visualization for statistics in social science. $R$ Package

$514 \quad$ Version, 2(0).

Madeira, N., \& Oliveira, R. F. (2017). Long-term social recognition memory in zebrafish.

Miklosi, A., Csanyi, V., \& Gerlai, R. (1997). Antipredator behavior in paradise fish Zebrafish, 14(4), 305-310.

Müller, C. A., \& Manser, M. B. (2007). 'Nasty neighbours' rather than 'dear enemies' in a social carnivore. Proceedings of the Royal Society of London B: Biological Sciences, 274(1612), 959-965.

Nakahashi, W., \& Ohtsuki, H. (2015). When is emotional contagion adaptive? Journal of Theoretical Biology, 380, 480-488. https://doi.org/10.1016/j.jtbi.2015.06.014

525 Nakahashi, W., \& Ohtsuki, H. (2018). Evolution of emotional contagion in group-living animals. Journal of Theoretical Biology, 440, 12-20. 
527 Oliveira, T. A., Idalencio, R., Kalichak, F., dos Santos Rosa, J. G., Koakoski, G., de Abreu,

528 M. S., Giacomini, A. C. V., Gusso, D., Rosemberg, D. B. \& Barreto, R. E. (2017). Stress

529 responses to conspecific visual cues of predation risk in zebrafish. PeerJ 5, e3739.

$530 \quad$ https://doi.org/10.7717/peerj.3739

531 Pinheiro-da-Silva, J., Silva, P. F., Nogueira, M. B., \& Luchiari, A. C. (2016). Sleep

532 deprivation effects on object discrimination task in zebrafish (Danio rerio). Animal

533 Cognition, 1-11. https://doi.org/10.1007/s10071-016-1034-x

534 Pisansky, M. T., Hanson, L. R., Gottesman, I. I., \& Gewirtz, J. C. (2017). Oxytocin enhances

$535 \quad$ observational fear in mice. Nature Communications, 8(1), 2102.

536 https://doi.org/10.1038/s41467-017-02279-5

537 Preston, S. D., \& de Waal, F. B. M. (2002). Empathy: Its ultimate and proximate bases. The

$538 \quad$ Behavioral and Brain Sciences, 25(1), 1-20; discussion 20-71.

$539 \quad$ https://doi.org/10.1017/S0140525X02000018

540 R Core Team (2013). R: A language and environment for statistical computing. Vienna,

$541 \quad$ Austria: R Foundation for Statistical Computing.

542 Reimert, I., Bolhuis, J. E., Kemp, B., \& Rodenburg, T. B. (2013). Indicators of positive and

543 negative emotions and emotional contagion in pigs. Physiology and Behavior, 109(1),

$544 \quad$ 42-50. https://doi.org/10.1016/j.physbeh.2012.11.002

545 Reimert, I., Bolhuis, J. E., Kemp, B., \& Rodenburg, T. B. (2014). Emotions on the loose:

546 emotional contagion and the role of oxytocin in pigs. Animal Cognition, 18(2), 517-532.

$547 \quad$ https://doi.org/10.1007/s10071-014-0820-6

548 Rey, S., Huntingford, F. A., Boltaña, S., Vargas, R., Knowles, T. G., \& Mackenzie, S. (2015).

549 Fish can show emotional fever $\square$ : stress-induced hyperthermia in zebrafish. Proceedings 
Roberts, L. J., \& Garcia de Leaniz, C. (2011). Something smells fishy: predator-naïve salmon use diet cues, not kairomones, to recognize a sympatric mammalian predator. Animal

von Frisch, K. (1938). On the psychology of the fish shoal. Naturwissenschaften, 26, 601-

Swaney, W., Kendal, J., Capon, H., Brown, C., \& Laland, K. N. (2001). Familiarity facilitates

\section{Tran, S., \& Gerlai, R. (2013). Individual differences in activity levels in zebrafish (Danio} social learning of foraging behaviour in the guppy. Animal Behaviour, 62(3), 591-598. https://doi.org/10.1006/anbe.2001.1788 606. 